\title{
Novel Design of Ring Resonator Based Temperature Sensor Using Photonics Technology
}

\author{
Massoudi RADHOUENE ${ }^{1}$, Mayur Kumar CHHIPA ${ }^{2}$, Monia NAJJAR ${ }^{1,3}$, \\ S. ROBINSON ${ }^{4}$, and Bhuvneshwer SUTHAR ${ }^{5 *}$ \\ ${ }^{1}$ University of Tunis El Manar, National Engineering School of Tunis Communications Systems LR-99-ES21 \\ (LR-Sys 'Com-ENIT), Tunis, 1002, Tunisia \\ ${ }^{2}$ Department of Electronics \& Communication Engineering, K L University, Guntur, Andhra Pradesh, 522502, India \\ ${ }^{3}$ University of Tunis El Manar, Higher Institute of Computer, Ariana, 2080, Tunisia \\ ${ }^{4}$ Department of Electronics \& Communication Engineering, Mount Zion College of Engineering and Technology, \\ Pudukkottai, Tamil Nadu, 622507, India \\ ${ }^{5}$ Department of Physics, Govt. Engineering College, Bikaner, Rajasthan, 334004, India \\ *Corresponding author: Bhuvneshwer SUTHAR_Ｅ-mail: bhuvneshwer@gmail.com
}

\begin{abstract}
In the present paper, we study the transmission of the two-dimensional photonic crystal (PC) superellipse ring resonator. The fast growing applications of optomechanical systems lead to strong demands in new sensing mechanism in order to design the sensing elements to nanometer scale. The photonic crystal based resonator has been investigated as promising solutions because the band gap structure and resonator characteristics are extremely sensitive to the deformation and position shift of rod/cavity in PC resonators. This structure opens a single channel filter. The study is extended for tuning of channel filter's wavelength with a temperature of this structure. The transmission of the channel filter shows a red shift with temperature linearly. This wavelength shift of the channel filter is used for the sensor application. The sensitivity for the proposed structure is found to be $65.3 \mathrm{pm} /{ }^{\circ} \mathrm{C}$. The outstanding sensing capability renders $\mathrm{PC}$ resonators as a promising optomechanical sensing element to be integrated into various transducers for temperature sensing applications.
\end{abstract}

Keywords: Photonic crystal ring resonator; temperature sensor; finite difference time domain; PWE method

Citation: Massoudi RADHOUENE, Mayur Kumar CHHIPA, Monia NAJJAR, S. ROBINSON, and Bhuvneshwer SUTHAR, "Novel Design of Ring Resonator Based Temperature Sensor Using Photonics Technology," Photonic Sensors, 2017, 7(4): 311-316.

\section{Introduction}

Photonic crystal (PC) has the most important properties to confine and control the propagation of the light inside the crystal [1]. Based on this characteristic, many scientists focused their works to design and investigate various optical devices, such as optical filters [2-4], demultiplexers [5-11], circulators [12], power splitters [13], and switches [14].

This characteristic property of photonic crystals (PCs) has been explored a new field of research of optical sensing. The sensor is a device, which can detect and respond to some different inputs from the

Received: 7 June 2017 / Revised: 18 July 2017

(C) The Author(s) 2017. This article is published with open access at Springerlink.com

DOI: $10.1007 / \mathrm{s} 13320-017-0443-\mathrm{z}$

Article type: Regular 
physical environment. In recent years, the optical sensors have been attracted much attention of the community due to the advantage over electronic sensors. The electronic sensors have limitation to transfer the large data with higher speed, which can be solved by the tunable filter, all optical switches, and optical sensors [15-19]. The optical sensor is designed by using an optical fiber, a photonic crystal fiber, and photonic crystals [19, 20].

The most parameters can be characterized by using an optical sensor with the good value of quality factor and high sensitivity. In the past few years, many researchers designed the optical sensors by using the photonic crystal ring resonator (PCRR). In the literature, many researchers worked on the optical sensors for various sensing applications such as temperature [21], pressure [22], and bio-chemical [23-25] sensors.

Recently, C. Mallika et al. proposed a ring resonator based sensor for the detection of temperature [21], in which the value of wavelength shifted when applying an external effect in the whole structure. Robinson et al. designed an optical sensor based on the photonic ring resonator to detect the salinity with different temperatures. The simulation results showed a good value of quality factor and normalized transmission, which were equal to 477.83 and $99.5 \%$, respectively at the wavelength of $1590.55 \mathrm{~nm}$ with the temperature of $25^{\circ} \mathrm{C}$ [26].

In the present work, we showed that the wavelength at resonance was obtained at Port $\mathrm{C}$ to open a channel filter. The channel filter's wavelength can be tuned with the temperature of the device as an external parameter. The tuning of channel filter's wavelength can be used to design a photonic temperature sensor.

\section{Bandgap analysis}

In this section, our proposed structure of a 2-D photonic crystal based on a superellipse ring resonator is designed. We place the rods of
$\mathrm{In}_{0.53} \mathrm{Al}_{0.16} \mathrm{Ga}_{0.31} \mathrm{As}$, with a higher refractive index equal to 3.19 in the air (with a refractive index 1). The numbers of rods in $x$ and $z$ directions are 35 and 28 with a lattice constant of $546 \mathrm{~nm}$. So the device size will be $277 \mu \mathrm{m}^{2}$. In general, the photonic band gap depends on three parameters: the permittivity of dielectric materials, the radius of rods, and the spacing between two rods. The values of radius and the lattice constant are investigated by using the gap map diagram as shown in Fig. 1.

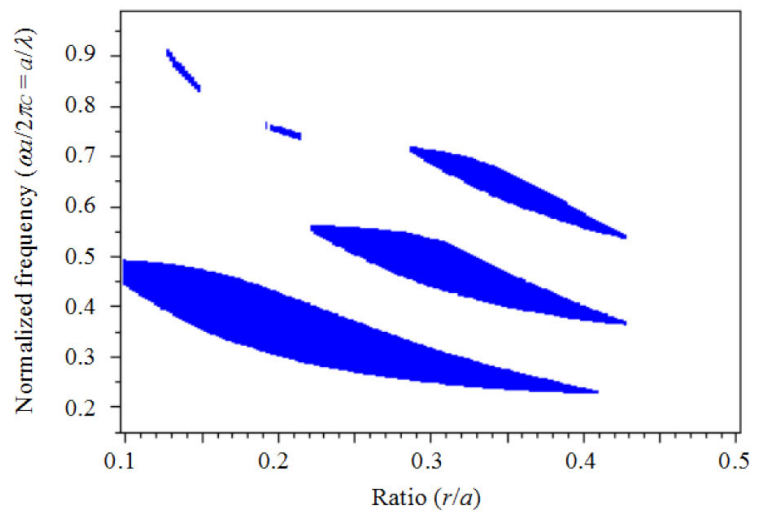

Fig. 1 Band gap map of the photonic crystal.

The band gap map diagram in the above figure presents the variation of the normalized frequency versus the ratio $(r / a)$ ( $r$ is the radius of dielectric rods, and $a$ is the spacing between two neighbor rods for the photonic crystal. According to Fig. 1, the maximum band width obtained at $r / a$ is equal to 0.185 . Therefore, corresponding to this ratio, the period $(a)$ is equal to $546 \mathrm{~nm}$, and the value of radius $(r)$ is to be set as $0.185 \times a \mathrm{~nm}$.

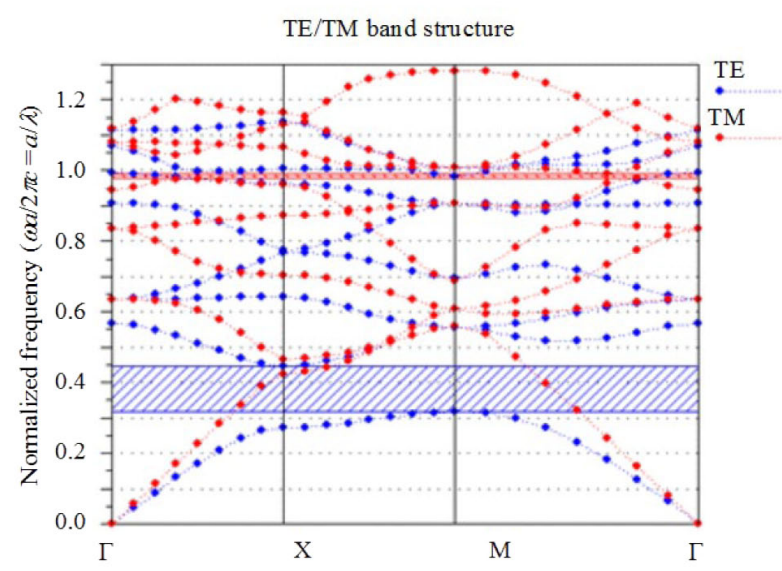

Fig. 2 Photonic band structure of the 2-D photonic crystal. 
The band structure is plotted by using the plane wave expansion method (PWE) [27] for the regular 2-D photonic crystal (2-D PC) without defect as shown in Fig. 2. For the above parameters for the PC, two photonic bandgap (PBG) regions in the band structure are achieved: the first region designated by the lower shaded region is in transversal electric (TE) mode, and the second region designated by the upper shaded region is in transversal magnetic (TM) mode.

The normalized frequency of the first region is between 0.32 and 0.45 and in the wavelength range from $1213.33 \mathrm{~nm}$ to $1706.25 \mathrm{~nm}$. On the other side, the second PBG region in TM is between 0.977 and 0.991 in the wavelength range from $0.55 \mathrm{~nm}$ up to $0.558 \mathrm{~nm}$, which is less suitable for our proposed design for sensing application in this range.

\section{Temperature sensor design}

In this section, our proposed structure is based on a 2-D PC superellipse ring resonator. We place the rods with a higher refractive index equal to 3.19 , in the air (with a refractive index 1). The numbers of rods in $x$ and $z$ directions are 35 and 28, respectively. The photonic bandstructure diagram is plotted for the radius of the rods $r=0.185 a$, where $a$ is the lattice constant, whose corresponding value has been taken as $546 \mathrm{~nm}$. All the designed parameters have been optimized as per sensing application.

Figure 3 shows the novel design of 2-D PC based on the superellipse ring resonator. To design the proposed structure, we create two parallel waveguides called as drop and bus waveguides, respectively as shown in Fig. 3. A novel superellipse ring is placed at the middle of the structure between bus and drop waveguides.

Our proposed sensor consists of three outputs:

(1) Port A: as forward transmission port placed at the end of bus waveguide;

(2) Port B: as forward drop placed at the extreme end of drop waveguide in the right;

(3) Port C: as backward drop placed at the extreme end of drop waveguide in the left.

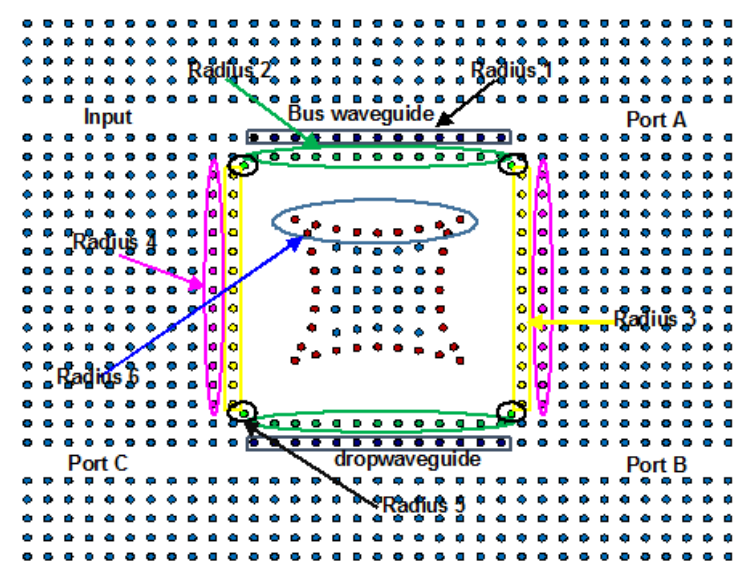

Fig. 3 Schematic diagram of the proposed superellipse ring resonator structure.

In this design, specific rods (coupling, adjacent, and scatterer) with different radii are chosen to ameliorate their performances. Figure 3 shows six colored dots representing different radii of this structure. The first step of this work is to optimize those radii. The values of Radius 1 to Radius 6 are achieved after a number of iterations. The optimized values of radii of rods for each row are obtained by varying the radius between the minimum and maximum limits, i.e. starting from zero, up to a maximum value of $0.5 \times a$ of the corresponding row. The optimized radius values for different rods are listed in Table 1.

Table 1 Optimized value of radius of different dielectric rods.

\begin{tabular}{cc}
\hline Rods & Radius (nm) \\
\hline Radius 1 & $0.185 a$ \\
Radius 2 & $0.450 a$ \\
Radius 3 & $0.185 a$ \\
Radius 4 & $0.185 a$ \\
Radius 5 & $0.185 a$ \\
Radius 6 & $0.200 a$ \\
\hline
\end{tabular}

\section{Results and discussion}

In this section, the transmission and propagation of electromagnetic light wave through the structure are simulated by using the finite difference time domain (FDTD) method [28]. The perfectly matched 
layers (PML) boundary conditions are assumed with the width equal to $500 \mathrm{~nm}$. The output transmissions for the proposed structure for various ports A, B, and $\mathrm{C}$ are obtained, as shown in Fig. 4.

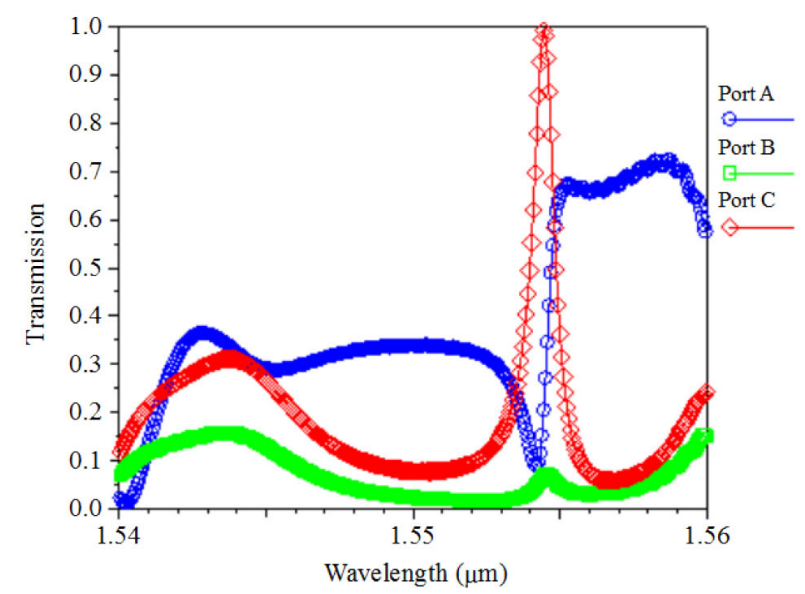

Fig. 4 Transmission for the proposed 2-D photonic crystal superellipse ring resonator PCSRR structure for Ports A, B, and $\mathrm{C}$.

According to Fig. 4, the resonance wavelength equal to $1554.5 \mathrm{~nm}$ is dropped and guided to Port C. The signal will be received at output port $\mathrm{C}$ at about 3 ps. Therefore, this sensor device response is within $\sim$ ps or known as the ultrafast sensor. The most important parameter used to characterize the proposed design is the quality factor $(Q)=\lambda_{0} / \Delta \lambda$, where $\lambda_{0}$ is the resonance wavelength, and $\Delta \lambda$ is the wavelength width around $\lambda_{0}$ at the full wave half maximum (FWHM). Based on this formula, the value of quality factor of the given structure equals 1727 at the wavelength $1554.5 \mathrm{~nm}$.

The transmission at Port $\mathrm{C}$ shows a sharp peak corresponding to the resonance wavelength, which opens a channel to filter the input signal. On the other hand, the transmission at Port A shows a very sharp fall at the resonance wavelength, and the above resonance wavelength signal has a good transmission. The resonance wavelength works as a threshold wavelength, and this port can be used as an optical limiter.

The material refractive index varies with temperature as an external parameter [8]. This variation is expressed as (1):

$$
n=n_{0}+\gamma \Delta T
$$

where $n_{0}=3.19$ (refractive index value at $0{ }^{\circ} \mathrm{C}$ temperature), $\gamma=1.18 \times 10^{-3} /{ }^{\circ} \mathrm{C}$, and $\Delta T$ is the change in temperature.

As the temperature increases, the refractive index increases also, thus, the resonance wavelength is tuned. The transmission at Port $\mathrm{C}$ at various temperatures is plotted in Fig. 5 .

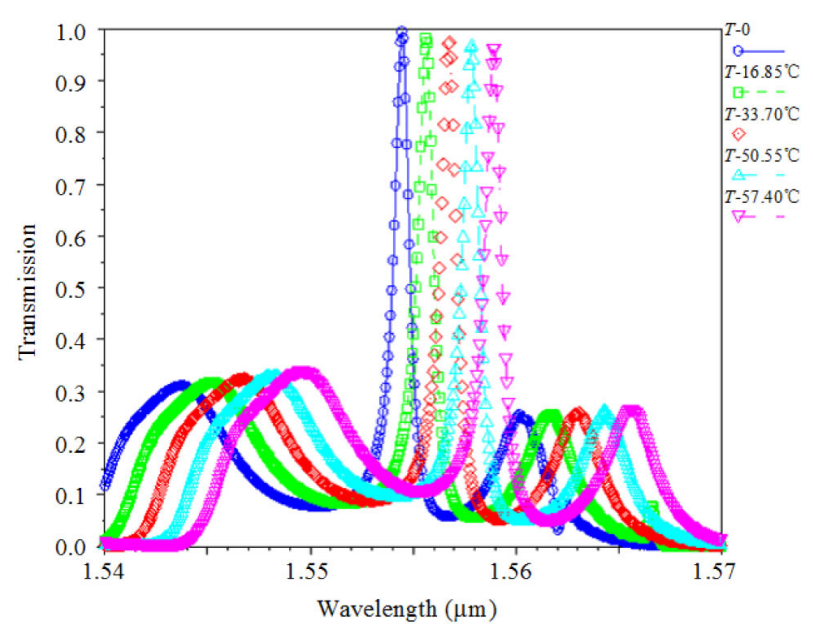

Fig. 5 Transmission at Port $\mathrm{C}$ at different temperatures $\left(16.85^{\circ} \mathrm{C}, 33.70^{\circ} \mathrm{C}, 50.55^{\circ} \mathrm{C}\right.$, and $\left.67.40^{\circ} \mathrm{C}\right)$.

From Fig. 5, it is clearly observed that the resonance wavelength shows a red shift (shifting towards the higher wavelength) with an increase in temperature. The wavelength at resonance is obtained at Port $\mathrm{C}$ to open a channel filter. The channel filter's wavelength can be tuned with the temperature of the device as an external parameter. The tuning of channel filter's wavelength can be used to design a photonic temperature sensor.

Therefore, Port $\mathrm{C}$ can be used as a single channel filter which shows tuning with the temperature of the device. The values of wavelength and the quality factors at various temperatures are summarized in Table 2.

From Table 2, it is shown that the tuning of channel filter's wavelength shows a linear response with the temperature. The channel filter's wavelength is shifted $1.1 \mathrm{~nm}$ per $16.85{ }^{\circ} \mathrm{C}$ temperature change. Therefore, our proposed structure, depending on the linear properties of the 
tunable channel filter with temperature, can be used to develop the temperature sensor.

Table 2 Resonance wavelengths and quality factors at various temperatures.

\begin{tabular}{cccc}
\hline $\begin{array}{c}\text { Temperature } \\
\left({ }^{\circ} \mathrm{C}\right)\end{array}$ & $\begin{array}{c}\text { Central } \\
\text { wavelength }(\mathrm{nm})\end{array}$ & $\begin{array}{c}Q \text {-factor } \\
(Q)\end{array}$ & $\begin{array}{c}\text { Wavelength shift } \\
(\mathrm{nm})\end{array}$ \\
\hline 0.00 & 1554.5 & 1727.2 & -- \\
16.85 & 1555.6 & 1728.4 & 1.1 \\
33.70 & 1556.7 & 1556.7 & 1.1 \\
50.55 & 1557.8 & 1557.8 & 1.1 \\
67.40 & 1558.9 & 1558.9 & 1.1 \\
\hline
\end{tabular}

The sensor is characterized by an important parameter which is named sensitivity. The sensitivity for the temperature sensor is given as follows:

$$
S=d \lambda / d T \text {. }
$$

The sensitivity parameter shows the wavelength shift per unit temperature, which means the larger wavelength shift with temperature shows the good sensitivity for the device. In the present case, the sensitivity of the proposed 2-D PCSRR sensor is $0.0653 \mathrm{~nm} /{ }^{\circ} \mathrm{C}$ or $65.3 \mathrm{pm} /{ }^{\circ} \mathrm{C}$.

\section{Conclusions}

In this paper, the transmission of the 2-D PC superellipse ring resonator is studied. The functional parameters, namely resonant wavelength, $Q$ factor, channel spacing, spectral width, wavelength shift, and sensitivity, are investigated. The result shows the single channel filter with the wavelength $1554.5 \mathrm{~nm}$ and quality factor 1727.22 . The channel filter's wavelength shows a red shift with an increase in the temperature. This tunable channel filter shows the wavelength shifts linearly with the temperature. The linear response of channel filter's wavelength shift is used as a temperature sensor with a temperature sensitivity $65.3 \mathrm{pm} /{ }^{\circ} \mathrm{C}$.

Open Access This article is distributed under the terms of the Creative Commons Attribution 4.0 International License (http://creativecommons.org/ licenses/by/4.0/), which permits unrestricted use, distribution, and reproduction in any medium, provided you give appropriate credit to the original author(s) and the source, provide a link to the Creative Commons license, and indicate if changes were made.

\section{References}

[1] J. D. Joannopoulos, S. G. Johnson, J. N. Winn, and R. D. Meade, Photonic crystals: molding the flow of light. New Jersey, USA: Princeton University Press, 2011: 38-47.

[2] S. Robinson and R. Nakkeeran, "Investigation on two dimensional photonic crystal resonant cavity based bandpass filter," Optik-International Journal for Light and Electron Optics, 2012, 123(5): 451-457.

[3] H. Alipour-Banaei and F. Mehdizadeh, "A proposal for anti-UVB filter based on one-dimensional photonic crystal structure," Digest Journal of Nanomaterials and Biostructures, 2012, 7(1): 361-371.

[4] M. Radhouene, M. Najjar, and R. Houria, "Optimization of WDM demultiplexer based on photonic crystal," in 2015 World Symposium on Computer Networks and Information Security (WSCNIS), Hammamet, Tunisia, 2015, pp. 1-4.

[5] H. P. Bazargani, "Proposal for a 4-channel all optical demultiplexer using 12-fold photonic quasicrystal," Optics Communications, 2012, 285(7): 1848-1853.

[6] A. Rostami, F. Nazari, H. A. Banaei, and A. Bahrami, "Anovel proposal for DWDM demultiplexer design using modified-T photonic crystal structure," Photonics and Nanostructures-Fundamentals and Applications, 2010, 8(1): 14-22.

[7] S. C. Cheng, J. Z. Wang, L. W. Chen, and C. C. Wang, "Multichannel wavelength division multiplexing system based on silicon rods of periodic lattice constant of hetero photonic crystal units," Optik-International Journal for Light and Electron Optics, 2012, 123(21): 1928-1933.

[8] A. Rostami, H. A. Banaei, F. Nazari, and A. Bahrami, "An ultra compact photonic crystal wavelength division demultiplexer using resonance cavities in a modified Y-branch structure," Optik-International Journal for Light and Electron Optics, 2011, 122(16): 1481-1485.

[9] S. Rawal and R. K. Sinha, "Design, analysis and optimization of silicon-on-insulator photonic crystal dual band wavelength demultiplexer," Optics Communications, 2009, 282(19): 3889-3894.

[10] B. Momeni, J. Huang, M. Soltani, M. Askari, S. Mohammadi, M. Rakhshandehroo, et al., "Compact wavelength demultiplexing using focusing negative index photonic crystal superprisms," Optics Express, 2006, 14(6): 2413-2422. 
[11] P. Louro, M. Vieira, M. Fernandes, M. A. Vieira, J. Costa, and A. Fantoni, "Semiconductor device as optical demultiplexer for short range optical communications," Journal of Nanoscience Nanotechnology, 2011, 11(6), 5318-5322.

[12] Z. Wang and S. Fan, "Optical circulators in two-dimensional magneto-optical photonic crystals," Optics Letters, 2005, 30(15): 1989-1991.

[13] M. Bayindir, B. Temelkuran, and E. Ozbay, "Photonic-crystal-based beam splitters," Applied Physics Letters, 2000, 77(24): 3902-3904.

[14] T. A. Tameh, B. M. Isfahani, N. Granpayeh, and A. M. Javan, "Improving the performance of all-optical switching based on nonlinear photonic crystal microring resonators," AEU-International Journal of Electronics and Communications, 2011, 65(4): 281-287.

[15] H. Z. Wang, W. M. Zhou, and J. P. Zheng, “A 2-D rods-in-air square-lattice photonic crystal optical switch," Optik-International Journal for Light and Electron Optics, 2010, 121(21): 1988-1993.

[16] R. Massoudi, M. Najjor, and V. Janyani, "Tunable photonic crystal switch based on ring resonators with improved crosstalk and $Q$-factor," SPIE, 2017: 102500Z-1-102500Z-5.

[17] M. K. Chhipa, R. Massoudi, S. Robinson, and B. Suthar, "Improved dropping efficiency in two-dimensional photonic crystal-based channel drop filter for coarse wavelength division multiplexing application," Optical Engineering, 2017, 56(1): 015107-1-015107-6.

[18] B. Suthar, "Tuning of guided mode in two dimensional chalcogenide based photonic crystal waveguide," Optik-International Journal for Light and Electron Optics, 2015, 126(22): 3429-3431.

[19] I. Park, H. S. Lee, H. J. Kim, K. M. Moon, S. G. Lee, S. G. Park, et al., "Photonic crystal power-splitter based on directional coupling," Optics Express, 2004,
12(15): 3599-3604.

[20] V. Zabelin, L. A. Dunbar, N. L. Thomas, R. Houdré, M.V. Kotlyar, L. O'Faolain, et al., "Self-collimating photonic crystal polarization beam splitter," Optics Letters, 2007, 32(5), 530-532.

[21] C. S. Mallika, I. Bahaddur, P. C. Srikanth, and P. Sharan, "Photonic crystal ring resonator structure for temperature measurement," Optik-International Journal for Light and Electron Optics, 2015, 126(20): 2252-2255.

[22] K. V. Shanthi and S. Robinson, "Two-dimensional photonic crystal based sensor for pressure sensing," Photonic Sensors, 2014, 4(3): 248-253.

[23] P. Sharma and P. Sharan, "Photonic crystal based ring resonator sensor for detection of glucose concentration for biomedical applications," International Journal of Emerging Technology and Advanced Engineering, 2014, 4(30): 702-706.

[24] S. Olyaee and A. Mohebzadeh-Bahabady, "Designing a novel photonic crystal nano-ring resonator for biosensor application," Optical and Quantum Electronics, 2015, 47(7): 1881-1888.

[25] K. P. Swain and G. Palai, "Estimation of human-hemoglobin using honeycomb structure: an application of photonic crystal," Optik-International Journal for Light and Electron Optics, 2016, 127(6): 3333-3336.

[26] S. Robinson and R. Nakkeeran, "PC based optical salinity sensor for different temperatures," Photonic Sensors, 2012, 2(2): 187-192.

[27] S. G. Johnson and J. D. Joannopoulos, "Block-iterative frequency-domain methods for Maxwell's equations in a planewave basis," Optics Express, 2001, 8(3): 173-190.

[28] S. D. Gedney, "Introduction to the finite-difference time-domain (FDTD) method for electromagnetics," Synthesis Lectures on Computational Electromagnetics, 2011, 6(1): 1-250. 